\title{
$\underline{\mathbf{P}-160}$
}

\section{Flavonoids Constituents of Four Varieties of Ficus deltoidea (Jack)}

\author{
Syazwani Dzolin ${ }^{1}$, Rohaya Ahmad ${ }^{1, *}$ and Mazatulikhma Mat Zain ${ }^{2}$ \\ ${ }^{I}$ Faculty of Applied Sciences, Universiti Teknologi MARA, 40450 Shah Alam, Malaysia; ${ }^{2}$ Institute of Science (IOS), \\ Universiti Teknologi MARA, 40450 Shah Alam, Malaysia; E-mail: rohayaahmad@salam.uitm.edu.my
}

Hierarchical cluster analysis (HCA) is a data-mining method, which reveals the basic structure of a data set and is used to perform classification on chromatographic data sets. A dendrogram is used to determine the merge of clusters by HCA whereby the branch length represents the distance between samples while the colour intensity represents the presence of metabolites. The higher the colour intensity, the more abundant the metabolites present. In this study, flavonoid constituents in aqueous leaves and figs extracts of four varieties of Ficus deltoidea (F.d) (Moraceae) namely var kunstleri (F.d.v.k), var angustifolia (F.d.v.a), var deltoidea (F.d.v.d) and var intermedia (F.d.v.i) were analyzed and characterized using liquid chromatography mass spectrometer quadrupole-time of flight (LCMS Q-TOF) via HCA technique. The HCA dendrogram revealed that the abundant flavonoids among the eight samples are epicatechin, quercetin-3-rutinoside, quercetin 5,4'-di- $O$-beta- $D$-glucopyranoside, myricetin and naringenin. Our study found that the distribution of the flavonoids differed between the four varieties and varied within the plant parts of each variety. To date, no comparative study on the flavonoid constituents of the different plant parts of the four varieties has been documented.

Keywords: Hierarchical Cluster Analysis (HCA), dendrogram, Liquid Chromatography Mass Spectrometer Quadrupole-Time of Flight (LCMS Q-TOF), Ficus deltoidea. 